\title{
Special design of removable partial denture restoration following surgical resection of mouth floor neoplasm
}

\begin{abstract}
This clinical report describes a special removable partial denture design and fabrication in the mandibular teeth rehabilitation of a patient diagnosed with carcinoma in the mouth floor. Following surgical resection of partial teeth and mandible, a severe scar adhesion between tongue and mouth floor tissue occurred, which was difficult to reconstruct the mandible and teeth defect with removable partial denture. A specific restoration design with clasps on abutment teeth lingual side and denture base on external oblique ridge, which can effectively escape from the adhesive area, was indicated and discussed.
\end{abstract}

Volume 6 Issue 3 - 2017

\author{
Yan Dong,' Wang XM, ${ }^{2}$ Gao B' \\ 'Department of Prosthetic Dentistry, Zhejiang University, China \\ ${ }^{2}$ Department of Oral Surgery, Hangzhou First People's Hospital, \\ China \\ Correspondence: Dong Yan, Department of Prosthetic \\ Dentistry, Zhejiang University, 88, jiefang Road, hang zhou, China, \\ Tel 057I-877846I9, Fax 057|-87767078, \\ Email yandong_66@163.com
}

Received: September 17, 2016 | Published: February 13, 2017

\section{Introduction}

Patients suffered from floor of mouth tumors or trauma after operation always become limited oral opening or/and tongue moving, because of vestibular groove fading away and scar adhesion. All above produce really a great difficulty for restoration of the mandibular dentition defect. This report presents a case of making removable partial dentures for a patient whose tongue and mouth floor was adhesive after tumor resection. A specific restoration design which can effectively escape from the adhesive area was indicated.

\section{Case report}

The patient was about 50-year-old, male. His chief compliant was to restore dentition defect. One year ago, he had tumor and partly mandible resection and his anterior teeth and premolar teeth of mandible were also removed due to carcinoma. On oral inspection, the soft tissues of mouth floor and tongue have severe scar adhesion, resulted in limited tongue moving and speaking clearly. Meanwhile, his posterior teeth incline lingual obviously and his tongue was hypertrophy with visible pressing impressions at edge of tongue by lower molar teeth. Moreover, his remaining alveolar ridge was low and narrow. Maxillary central incisors, lateral central incisors and left canine were remaining.

\section{Denture design}

The patient demanded removal partial denture to restore teeth defect. On maxillary, his posterior teeth were missing, which once had born the mainly function and aesthetics restoration, so a wider palatal bar was choose as the major connector and an aesthetic clasp was set on the labial surface on right lateral incisors and left canine, and occlusal rests was on left canine. For mandible and teeth loss, restoration with removal denture was difficult. As usual, such this kind of teeth defect without scar adhesion, denture base plate was placed on the lingual side and clasp was on buccal side because lingual flange region provides denture base enough extension. In this case, soft tissue adhesion with tongue and flange region was disappearing. So, all the remaining teeth should be taken full advantage of providing the retention and stability to the mandibular denture. In addition, elastic clasp was set on the lingual side and denture base plate was placed on the buccal side, which can effectively escape from the adhesive area. Segmented impression making (The first time impression) The patient's tongue and mouth floor have scar adhesion; leading to the normal impression tray cannot be placed parallel into the lingual side area of the remaining lower teeth at same time. So segmented impression method, which use two half-trays to get the left and right side dentition impression respectively, was chosen with individualization tray. Super-hard Type IV stone model was reproduced.

\section{Denture base plate fabrication}

On the two half-casts, heat-curing resin base plate (methyl methacrylate) was fabricated with necessary and enough extent to oral vestibule. The clasp was set on the lingual side of remaining lower teeth. Base plate with lingual side clasp was fitted in patient mouth for both sides. Maximum intercuspation position recording after fitting, the two half base plates were connected in mouth accurately by coldcuring resin (methyl methacrylate). Then, occlusal wax was used to record the maximum intercuspation position and transferred to a simulator. Artificial teeth were setting-up according to the occlusal recording.

\section{The second time impression making}

Using a complete normal tray, the second impression, which include artificial teeth, wax denture base and lingual side clasp, was made. The height diameter of tray need not higher than denture base, if month impossible opening widely, because the denture base plate has already enough extent. Then, we made super hard stone cast. The other steps, such as flasking and investing, were as usually. 


\section{Result}

The patient feels the denture has good retention, when chewing, he found it stable and balance without obvious pressing pain on mucosa.

\section{Discussion}

When mandibular anterior and premolars teeth are missing, usually, denture base plate is placed on the lingual side of the remaining tooth. However, this patient's floor of mouth becomes shallow and weak, which limits lingual denture base extending. Lingual mucosa is too thin to support dentures and transmit the occlusal force. Meaning while, patient posterior teeth on both side inclined lingual obviously leading it difficult for denture to try-in if base plate is placed on lingual side. We, therefore, designed the denture elastic clasp was set on the lingual side, which can make full use of the undercut, and denture base plate was placed on the buccal side to prevent concentration of occlusal stress on the thin mucosa. In addition, the area between the labial side of the lower jaw alveolar crest and external oblique ridge is characterized by thick cortex bone. Therefore, consideration was given to making use of these anatomical stronger support occlusal force areas in a prosthetic manner. Furthermore, for the patients whose tongue and mouth floor are adhesive after surgery, that makes conventional technology cannot achieve accurate impression of full dentition and defect area using one time impression. While segmented impression technology can be used effectively to solve this problem. But it needs an accurate connecting between the two-half permanent denture base plates in the mouth and the alignment of wax dikes also requires being accurate to achieve a satisfactory result of repair (Figure 1-8).

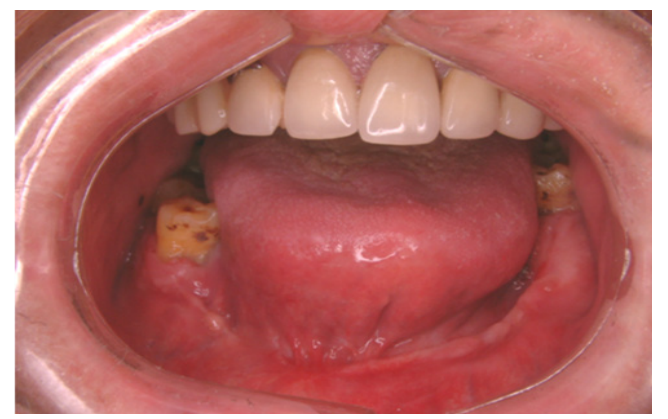

Figure I Intro-oral picture of patient after mouth floor tumor section. Soft tissues of mouth floor have scar adhesions with tongue severely, posterior teeth incline lingual obviously.

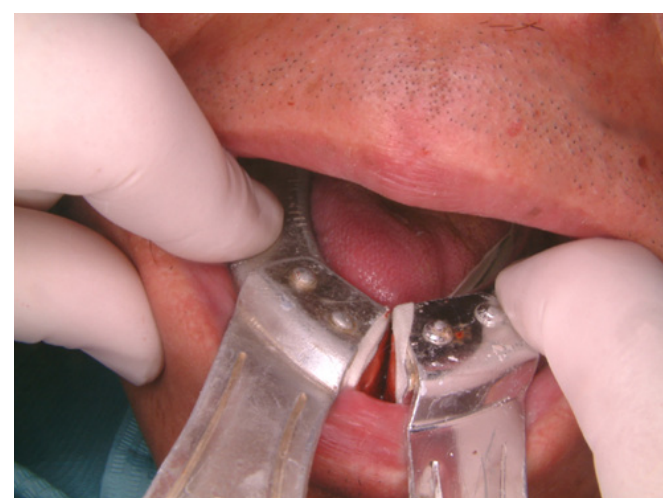

Figure 2 Segmented impression making. Two half-trays were used to get the left and right side dentition impression respectively.

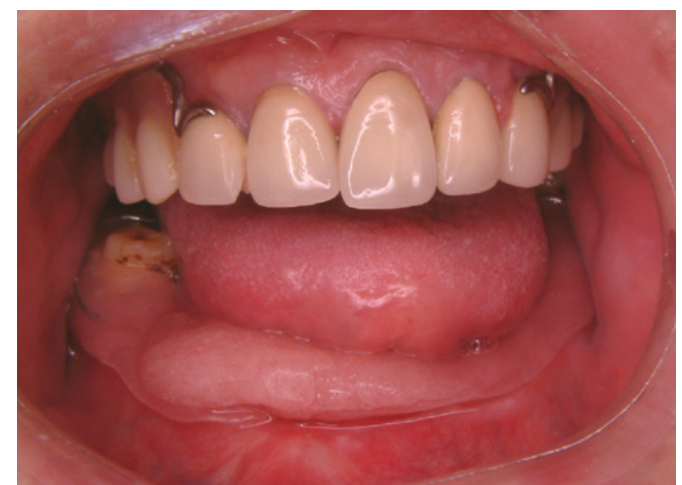

Figure 3 When fit, connect the left base plate with the right in the oral cavity.

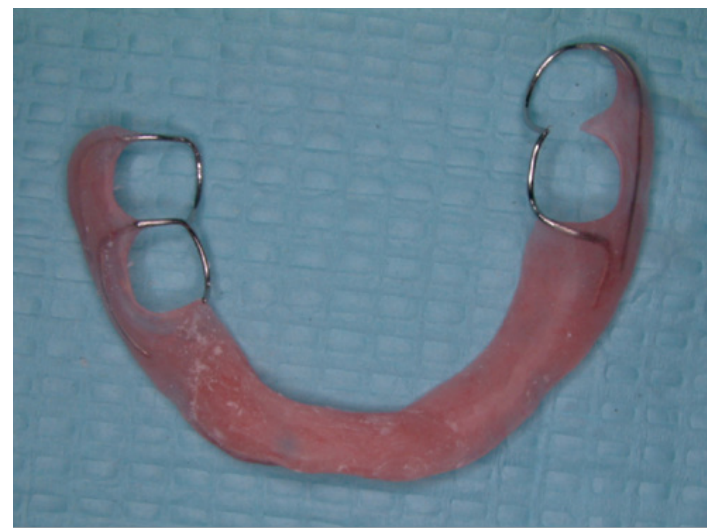

Figure 4 The appearance of the final base plate after connection and fitting.

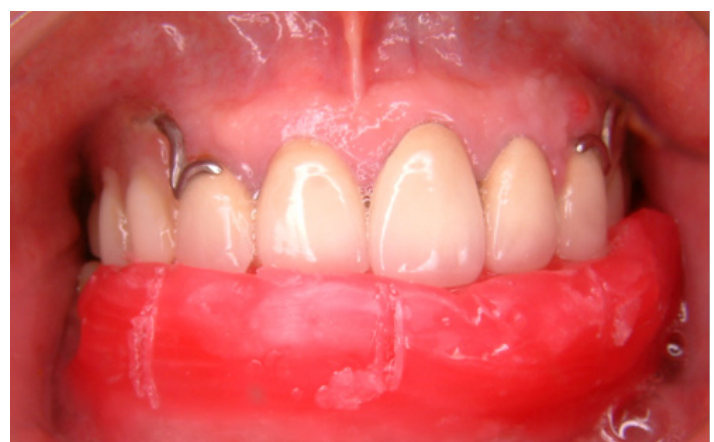

Figure 5 Occlusal wax was used to record the maximum intercuspation position in the patient's mouth.

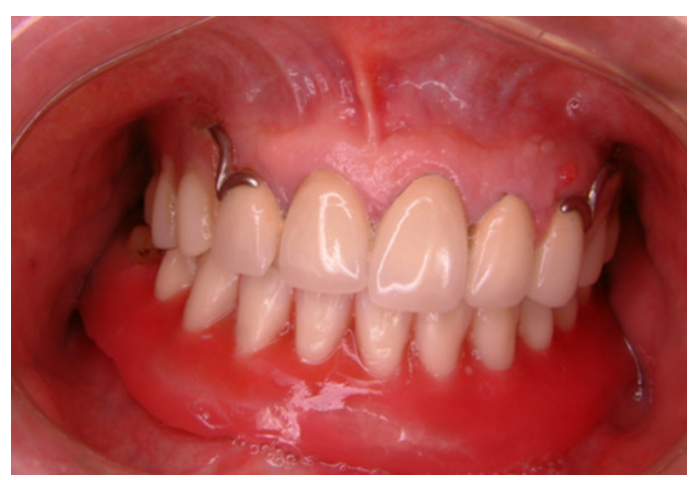

Figure 6 Try-in the denture after fitting. 


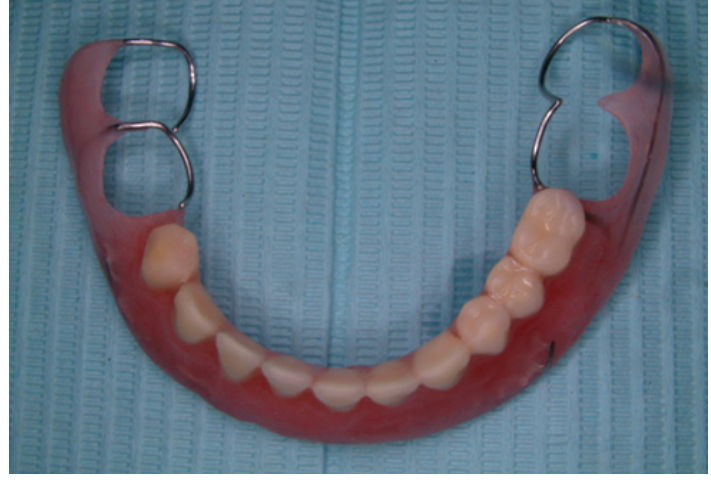

Figure 7 Removable partial denture with buccal base plate on external oblique line area and retention clasps on lingual side.

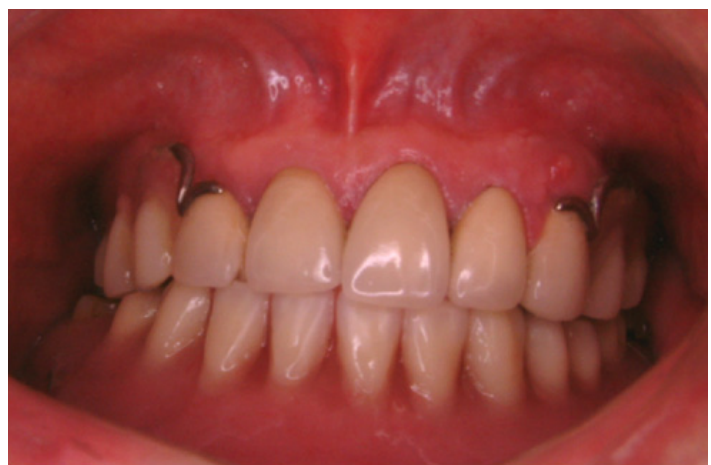

Figure $\mathbf{8}$ The anterior view of denture in the patient's mouth.

\section{Summary}

To sum up, this untraditional denture design helps patient avoid occlusal pressing pain and get better denture retention. ${ }^{1-3}$

\section{Funding}

None.

\section{Acknowledgements}

The authors gratefully acknowledge Mrs. Chen Min, for her valuable denture fabrication.

\section{Conflicts of interest}

The authors declare that there is no conflict of interest.

\section{References}

1. Kosaka M, Matsuzawa Y, Mori H, et al. Orbital wall reconstruction with bone grafts from the outer cortex of the mandible. $J$ Craniomaxillofac Surg. 2004;32(6):374-380.

2. Güngörmüș M, Yilmaz AB, Ertaş U, et al. Evaluation of the mandible as an alternative autogenous bone source for oral and maxillofacial reconstruction. J Int Med Res. 2002;30(3):260-264.

3. Li KK, Schwartz HC. Mandibular body bone in facial plastic and Reconstructive surgery. Laryngoscope. 1996;106(4):504-506. 